\title{
4,21-Dehydrogeissoschizine, an Intermediate in Heteroyohimbine Alkaloid Biosynthesis
}

By Martina Rueffer, Christiane Kan-Fan, $†$ Henri-Phillipe Husson, $\dagger$ Joachim Stöckigt, and Meinhart H. Zenk* (Lehrstuhl für Pflanzenphysiologie, Ruhr-Universität Bochum, D 4630 Bochum, W.-Germany, and $\dagger$ Institut de Chimie des Substances Naturelles, CNRS, F-91190 Gif-sur-Yvette, France)

Summary 4,21-Dehydrogeissoschizine (5) was proven to be a metabolic intermediate in the enzymatic synthesis of cathenamine (6) and its transformation products ajmalicine (7), 19-epiajmalicine (8), and tetrahydroalstonine (9); the enzyme catalysing the synthesis of (6) from (5) was identified and named cathenamine synthase.
THE reaction sequence leading from tryptamine (1) and secologanin (2) to heteroyohimbine alkaloids has largely been elucidated using cell-free systems from Catharanthus roseus. ${ }^{1-3}$ Besides strictosidine (3), a further intermediate in this pathway, cathenamine (6) $(20,21$-didehydroajmalicine; has been discovered.4 Furthermore Guettarda eximia (Rubiaceae) proved to be a rich source for this natural 
intermediate. $^{5}$ From the same plant material a second compound was isolated and its structure identified as 4,21-dehydrogeissoschizine ${ }^{6}(5)$. Chemical transformations ${ }^{6}$ of this compound lead to either cathenamine (6) or geissoschizine $(\mathbf{1 0})$. These biomimetic reactions reflect closely the postulated biosynthetic expectations of an intermediate located between 4,21-dehydrocorynantheine aldehyde (4) and cathenamine (6). 4,21-Dehydrogeissoschizine (5) was therefore tested as a substrate for heteroyohimbine alkaloid formation using enzyme preparations from $C$. roseus cell cultures in the presence of NADPH. ${ }^{7}$ Under these conditions $[0.2 \mu \mathrm{mol}$ of $(5), 1.0 \mu \mathrm{mol}$ NADPH, 50 $\mu \mathrm{mol} \mathrm{KPO}_{4}{ }^{2-}$ buffer, $\mathrm{pH} \mathbf{7} \cdot 0,1 \mathrm{mg}$ protein, total vol. 0.5 $\mathrm{ml}, 30^{\circ} \mathrm{C}, 120 \mathrm{~min}$ ] transformation of this substrate $(5)$ to (7) $(25 \%),(8)(18 \%)$, and (9) $(11 \%)$ was achieved. The products were analysed by quantitative t.l.c. followed by mass spectroscopy (each compound: $m / e 352 \mathrm{M}^{+}$) as well as radioimmunoassay. ${ }^{8}$ Omission of NADPH led to the formation of $(6)$ in $74 \%$ yield. Cathenamine (6) was identified by mass spectroscopy $\left(350 \mathrm{M}^{+}\right)$and quantified by conversion ${ }^{5}$ into (9) using $\mathrm{BH}_{4}^{-}$and subsequent radioimmunological determination. ${ }^{8}$ Employing this assay system the conversion of (5) into (6) was further characterized. The reaction proceeded linearly with time, and was affected by $\mathrm{pH}$ (maximal conversion at $\mathrm{pH} \mathbf{6 \cdot 5}$ ), and protein (saturation at $\left.3 \mathrm{mg} \mathrm{ml}^{-1}\right)$ and substrate concentration $\left(K_{\mathrm{m}} \quad 0.6\right.$ $\mathrm{mm})$. In the absence of enzyme no conversion of (5) into (6) occurred even after prolonged incubation up to $24 \mathrm{~h}$.

In order to establish (5) as an obligatory intermediate in the biosynthetic sequence leading to the heteroyohimbine alkaloids, $\left[1^{-14} \mathrm{C}\right]-(\mathbf{1})$ and $(2)$ were allowed to be transformed in the presence of a tenfold [with respect to (1)] excess of unlabelled (5), enzyme, and NADPH. The specific activity of the alkaloids $(\mathbf{7}),(\mathbf{8})$, and (9) formed was ten times less than that of the control experiment which was performed in the absence of (5). This proves that $(\mathbf{1})$ is channelled through (5) in the course of the formation of $(7),(8)$, and (9). Furthermore, short-term incubations of $\left[1-{ }^{14} \mathrm{C}\right]-(1)$ and (2) in the presence of $(5)$, led to the accumulation of radioactivity in (5). Reduction of labelled (5), in the presence of a carrier, by $\mathrm{BH}_{4}^{-}$gave radioactive isositsirikine (11a) and its $\mathrm{C}-16$ isomer $(\mathbf{1 1 b}) .^{6}$ This experiment proves that the suspected intermediate is formed under cell-free conditions from distant precursors and establishes (5) as an obligatory intermediate in the formation of the heteroyohimbine alkaloids (7), (8), and (9). The new enzyme described here, which catalyses the formation of (6) from (5), is named cathenamine synthase.

Under NADPH regenerating conditions [isocitrate dehydrogenase system] (5) is transformed by $C$. roseus extracts in ca. $10 \%$ yield into geissoschizine $(\mathbf{1 0})$ which is known not to be a direct precursor of (7) and its isomers ${ }^{2}$ (8) and (9).

If the sequence of reactions from (1) and (2) to the heteroyohimbine derivatives (7), (8), and (9) is allowed to proceed in the presence of $\mathrm{D}_{2} \mathrm{O}$ a total of two deuterium atoms is incorporated, one on to each of C-18 and C-20, respectively. ${ }^{9}$ In contrast, if (5) is used as substrate for the NADPHdependent reaction only one deuterium atom is incorporated into (7), (8), and (9). This result indicated that under these conditions no isomerisation takes place when (5) is reached which would lead to (4) or its 20, 21-isomer. Furthermore, the incorporation of only one deuterium atom during the conversion of $(5)$ into $(7),(8)$, and (9) supports the finding that (5) is the immediate precursor of (6) in the biosynthetic pathway as outlined in the Scheme.

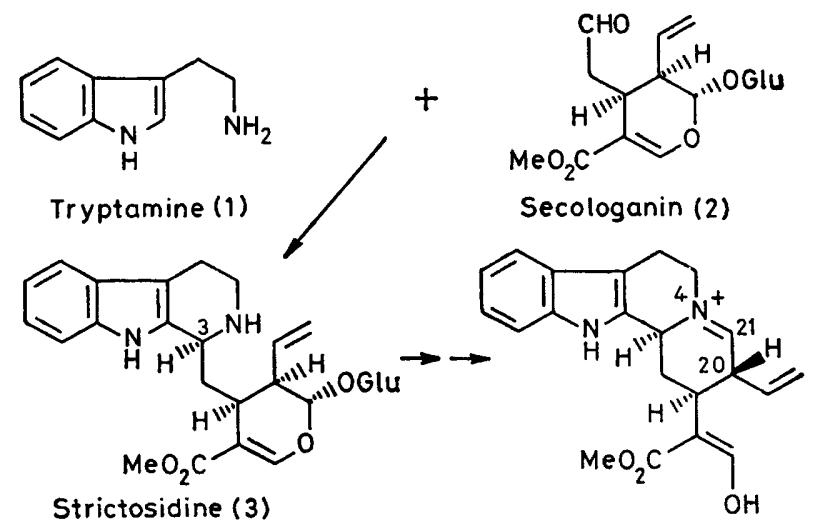

Strictosidine (3)

4,21-Dehydrocorynantheine aldehyde (4)

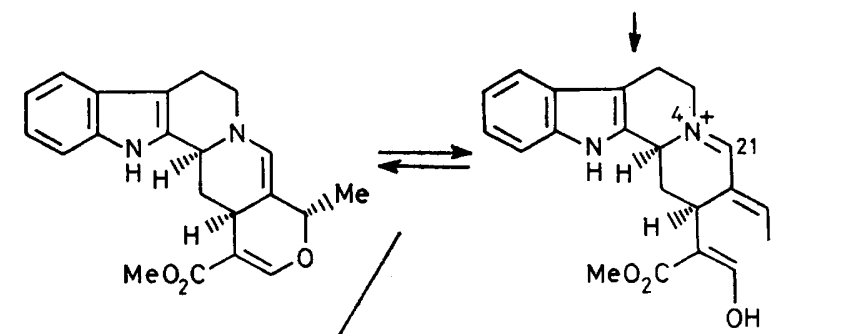

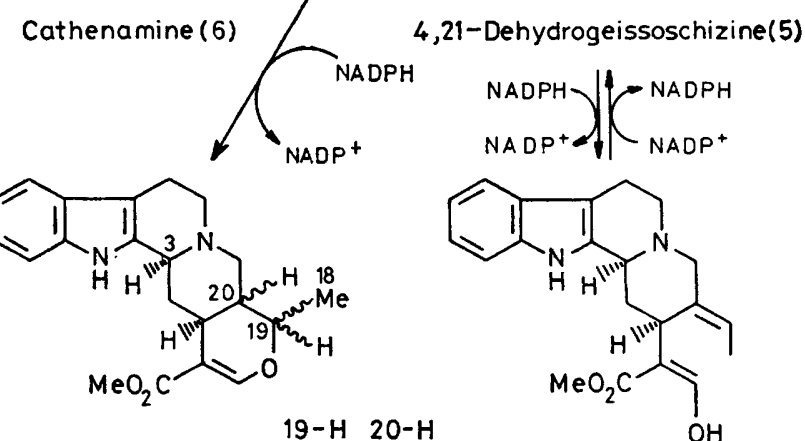

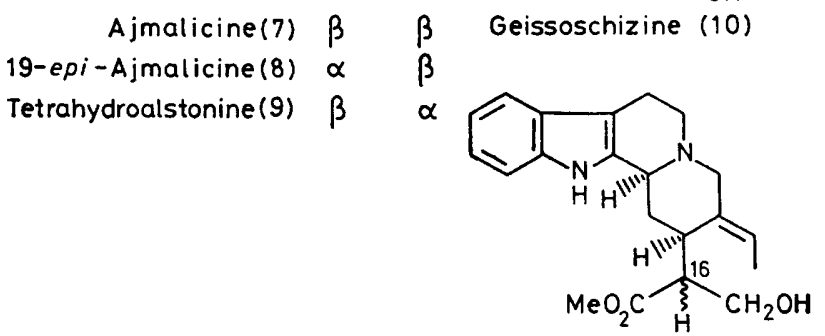

Isositsirikine $(16-H, \beta)(11 a)$ 16-epi-Isositsirikine $(16 H, \alpha)(11 b)$

SCHEME

We consider (5) to occupy a crucial position at the branch point in the biosynthesis of Iboga, Aspidosperma, and Corynanthe type alkaloids. 
We thank Mrs. W. Conrad and Miss A. Roder for excellent und Technologie, Bonn, for support. technical assistance, and the Bundesminister für Forschung

(Received, 3rd July 1979; Com. 717.)

1 J. Stöckigt, M. Rueffer, M. H. Zenk, and G.-A. Hoyer, Planta medica, 1978, 33, 188.

2 J. Stöckigt, J.C.S. Chem. Comm., 1978, 1097.

$3 \mathrm{~J}$. Stöckigt in, 'Indole and Biogenetically Related Alkaloids,' eds. J. D. Phillipson and M. H. Zenk, Academic Press, London, in the press.

4 J. Stöckigt, H.-P. Husson, C. Kan-Fan, and M. H. Zenk, J.C.S. Chem. Comm., 1977, 164.

5 H.-P. Husson, C. Kan-Fan, T. Sevenet, and J. P. Vidal, Tetrahedron Letters, 1977, 1889.

- C. Kan-Fan and H.-P. Husson, J.C.S. Chem. Comm., preceeding communication.

7 J. Stöckigt, J. Treimer, and M. H. Zenk, F.E.B.S. Letters, 1976, 70, 267.

${ }^{8} \mathrm{H}$. Arens, J. Stöckigt, E. W. Weiler, and M. H. Zenk, Planta medica, 1978, 34, 37.

J J. Stöckigt and P. Heinstein, to be published. 